\title{
Heterointegration of InGaAs/GaAs quantum wells on micro-patterned Si substrates
}

Cite as: J. Appl. Phys. 118, 075701 (2015); https://doi.org/10.1063/1.4928609

Submitted: 08 May 2015. Accepted: 03 August 2015 . Published Online: 17 August 2015

A. Jung, A. G. Taboada, W. Stumpf, T. Kreiliger (D, F. Isa, G. Isella, E. Barthazy Meier, and H. von Känel
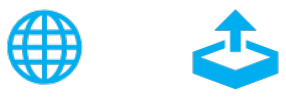

\section{ARTICLES YOU MAY BE INTERESTED IN}

GaAs/Ge crystals grown on Si substrates patterned down to the micron scale Journal of Applied Physics 119, 055301 (2016); https://doi.org/10.1063/1.4940379

III/V nano ridge structures for optical applications on patterned $300 \mathrm{~mm}$ silicon substrate Applied Physics Letters 109, 091101 (2016); https://doi.org/10.1063/1.4961936

Strain relaxation of GaAs/Ge crystals on patterned Si substrates

Applied Physics Letters 104, 022112 (2014); https://doi.org/10.1063/1.4861864

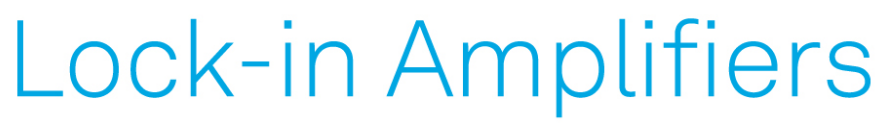

... and more, from DC to $600 \mathrm{MHz}$

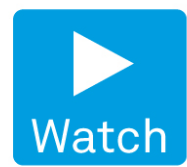




\title{
Heterointegration of InGaAs/GaAs quantum wells on micro-patterned Si substrates
}

\author{
A. Jung, ${ }^{1, a)}$ A. G. Taboada, ${ }^{1, b)}$ W. Stumpf, ${ }^{1}$ T. Kreiliger, ${ }^{1}$ F. Isa, ${ }^{1,2}$ G. Isella, ${ }^{2}$ \\ E. Barthazy Meier, ${ }^{3}$ and $\mathrm{H}$. von Känel ${ }^{1}$ \\ ${ }_{1}^{1}$ Laboratory for Solid State Physics, ETH Zürich, Otto-Stern-Weg 1, CH-8093 Zürich, Switzerland \\ ${ }^{2}$ L-NESS, Department of Physics, Politecnico di Milano, via Anzani 42, I-22100 Como, Italy \\ ${ }^{3}$ Scientific Center for Optical and Electron Microscopy (ScopeM), ETH Zürich, Auguste-Piccard-Hof 1, \\ CH-8093 Zürich, Switzerland
}

(Received 8 May 2015; accepted 3 August 2015; published online 17 August 2015)

\begin{abstract}
InGaAs/GaAs quantum wells (QWs) grown on $\mu$-patterned $\mathrm{Ge} / \mathrm{Si}$ substrates by metal organic vapor phase epitaxy are investigated by electron microscopy and spatially resolved photoluminescence (PL) spectroscopy. The lattice parameter mismatch of GaAs and $\mathrm{Si}$ is overcome by a Ge buffer layer grown by low-energy plasma enhanced chemical vapor deposition. The GaAs crystals form truncated pyramids whose shape is strongly affected by the geometry of the underlying pattern consisting of $8 \mu \mathrm{m}$ deep and 3-50 $\mu \mathrm{m}$ wide square Si pillars. Comparing the measured PL energies with calculations performed in the effective mass approximation reveals that the QW emission energies are significantly influenced by the GaAs morphology. It is shown that the geometry favors indium diffusion during growth from the inclined facets towards the top (001) facet. The Si pillarsize dependent release of thermally induced strain observed in the PL measurements is confirmed by X-ray diffraction. (C) 2015 AIP Publishing LLC. [http://dx.doi.org/10.1063/1.4928609]
\end{abstract}

\section{INTRODUCTION}

III/V compounds such as gallium arsenide ( $\mathrm{GaAs}$ ) form the fundamental ingredients of optoelectronic devices due to their high carrier mobilities $\left(9200 \mathrm{~cm}^{2} \mathrm{~V}^{-1} \mathrm{~s}^{-1}\right.$ for GaAs at $300 \mathrm{~K}$ ), and direct band gap. On the other hand, silicon ( $\mathrm{Si}$ ) is the dominant material in semiconductor electronics fostered by unrivalled crystal perfection, decades of miniaturization, low cost, and unlimited abundance. Thus, combining the benefits of both materials would pave the way for the monolithic integration of GaAs elements like quantum emitters and optical switches with Si integrated circuits featuring future multifunctional semiconductor devices. ${ }^{1,2}$

However, this heteroepitaxial integration is complicated by several issues, most notably the large lattice and thermal mismatch of the materials in question $(4.1 \%$ and $120 \%$, respectively, for $\mathrm{GaAs}$ on $\mathrm{Si}$ at $300 \mathrm{~K}) .^{2}$ Plastic strain relaxation by dislocations is therefore inevitable when GaAs beyond some critical thickness is grown onto $\mathrm{Si}$. For layer thickness above a few microns, wafer bowing and crack formation may become additional obstacles. ${ }^{1}$ Among the extended defects, threading dislocations (TDs) are the most problematic since they propagate through the whole epilayer up to its surface, acting as trapping and recombination centers for charge carriers. ${ }^{3}$ TDs always accompany the misfit dislocations forming at the materials' interfaces during the strain relaxation. Anti-phase domain boundaries arising from the growth of a polar semiconductor on a nonpolar substrate

\footnotetext{
a) Author to whom correspondence should be addressed. Electronic mail: junga@phys.ethz.ch.

b) Present address: Camlin Technologies CH, Technoparkstr. 1, 8005 Zurich, Switzerland.
}

are also critical defects. These can, however, be avoided by using offcut $\mathrm{Si}$ substrates. ${ }^{4,5}$

Much effort has been undertaken in the past decades in order to resolve the drawbacks arising from the monolithic integration of III/V compounds on Si. Aspect ratio trapping and epitaxial necking have evolved as efficient techniques to filter TDs by terminating their propagation. ${ }^{6,7} \mathrm{We}$ chose to apply the recently developed method of 3D-heteroepitaxy that makes use of the epitaxial necking mechanism. ${ }^{8}$ In this approach, germanium (Ge) crystals are grown on top of $\mu \mathrm{m}$ sized Si pillars by low-energy plasma enhanced chemical vapor deposition (LEPECVD). ${ }^{9}$ The peculiarity of this approach is that a large fraction of TDs escape through the Ge sidewalls such that crack-free, high quality relaxed Ge crystals are obtained. ${ }^{10}$ Furthermore, wafer bowing is avoided. Since Ge and $\mathrm{GaAs}$ are almost lattice matched $(0.08 \%$ mismatch at $300 \mathrm{~K}$ ) and only slightly differ in thermal expansion, low defect GaAs can be obtained by overgrowing these Ge crystals. ${ }^{2}$

\section{EXPERIMENTAL}

In this work, we have investigated the PL from InGaAs/ $\mathrm{GaAs}$ quantum well structures grown on various $\mathrm{Ge} / \mathrm{Si}$ pillars. Offcut $\mathrm{Si}(001)$ substrates $\left(6^{\circ} \text { towards }[110]\right)^{5}$ were patterned by conventional photolithography and deep reactive ion etching by means of the Bosch process. ${ }^{11}$ The patterns consisted of $8 \mu \mathrm{m}$-tall square Si pillars 3 to $50 \mu \mathrm{m}$ wide. The Si pillars were capped with $2 \mu \mathrm{m}$ of epitaxial Ge by LEPECVD at the growth rate and temperature of $4.2 \mathrm{~nm} / \mathrm{s}$ and $500{ }^{\circ} \mathrm{C}$, respectively. After epitaxial growth, the Ge were crystal was in-situ cyclically annealed between $600^{\circ} \mathrm{C}$ and $800^{\circ} \mathrm{C}$ for six times at a pressure of $\sim 5 \times 10^{-7}$ mbar. The III-V heterolayers were grown by metal-organic vapor-phase epitaxy (MOVPE) at a reactor pressure of 100 mbar. First, a $0.3 \mu \mathrm{m}$ GaAs buffer 
layer followed by a $0.3 \mu \mathrm{m}$ thick short-period $\mathrm{AlAs} / \mathrm{GaAs}$ superlattice was grown at $680^{\circ} \mathrm{C}$ and $720^{\circ} \mathrm{C}$, respectively. On top of the superlattice, $1.7 \mu \mathrm{m}$ of GaAs containing a single $\mathrm{In}_{0.13} \mathrm{Ga}_{0.87} \mathrm{As} \mathrm{QW}$ of $10 \mathrm{~nm}$ nominal thickness was deposited at $680^{\circ} \mathrm{C}$ and $660^{\circ} \mathrm{C}$. The QW is located $0.2 \mu$ m below the top (001)-facet. Since the band gap energy of AlAs is larger than that of GaAs, the superlattice serves as a barrier for charge carrier diffusion towards the Ge buffer region. Thereby, the active layer above the superlattice is electronically decoupled from the GaAs/Ge interface. Structural characterization was carried out with a Zeiss ULTRA 55 scanning electron microscope (SEM) and a FEI Tecnai F30 scanning transmission electron microscope (STEM). High resolution $\mathrm{X}$-ray diffraction (XRD) was performed with $\mathrm{Cu} \mathrm{K} \alpha_{1}$ radiation using a PANalytical X'Pert Pro-MRD diffractometer equipped with a 4-bounce $\mathrm{Ge}(220)$ crystal monochromator on the incident beam, and an analyzer crystal and a Xe point detector on the diffracted beam. The scattering plane was chosen perpendicular to the offcut direction. Spatially resolved PL was excited at $\mathrm{T}=5 \mathrm{~K}$ in a helium flow cryostat using a $638 \mathrm{~nm}$ continuous wave diode laser and a long working distance $100 \times$ near infrared microscope objective lens $(\mathrm{NA}=0.7$ ) yielding a focal spot size $\sim 2 \mu \mathrm{m}$. The spatial position of the collected PL relative to the sample was controlled by means of an optical camera. The PL spectra were taken with a liquid nitrogen cooled silicon charge coupled device camera attached to a TRIAX 550 spectrometer.

\section{RESULTS AND DISCUSSION}

\section{A. Electon micrographs}

Electron microscopy images of characteristic structures are presented in Figure 1. Perspective view SEM images of GaAs/Ge crystals on $5 \times 5 \mu \mathrm{m}^{2} \mathrm{Si}$ pillars (Figure 1(a)) illustrate the truncated pyramidal morphology. Bright field STEM images and the corresponding schematic view visualize the build-up of the heterolayers (Figure 1(b)). The Ge layer is located on top of the Si pillar, followed by the GaAs. The darker layer in the GaAs represents the $\mathrm{AlGaAs} / \mathrm{GaAs}$ superlattice. The InGaAs QW is visible as a faint, narrow white line below the top surface in the STEM picture. From the STEM analysis, we found a QW thickness of $16 \mathrm{~nm}$, $13 \mathrm{~nm}$, and $11 \mathrm{~nm}$ for GaAs/Ge on $3 \times 3 \mu \mathrm{m}^{2}, 5 \times 5 \mu \mathrm{m}^{2}$ and $15 \times 15 \mu \mathrm{m}^{2}$ pillars, respectively. However, the GaAs layer thickness slightly decreases with decreasing Si pillar dimensions, as inferred from the cross sections. The inclined surfaces of the pyramids are formed by $\{111\}$ and $\{113\}$ facets which are characterized by a significantly smaller growth rate compared to the (001) facet. ${ }^{12}$ The high stability of the GaAs $\{111\}$ surfaces favors the growth of the (001) surface, resulting in a truncated pyramidal crystal structure. ${ }^{13-15}$ Therefore, the pyramidal morphology of the GaAs depends on the size of the Si pillar as evidenced from the top view SEM images (Figures 1(c)-1(h)). A detailed description of the morphology can be found in the work of Taboada et al. ${ }^{2}$

\section{B. Photoluminescence}

Figure 2 shows the low temperature PL spectra measured by exciting the central area of the top (001) surface of the GaAs/Ge crystals on differently sized Si pillars. The peak centered at $\sim 1.5 \mathrm{eV}$ stems from recombination in the $\mathrm{GaAs}$, whereas the distinct peak on the lower energy side is attributed to emission from the InGaAs QW. The InGaAs emission energy gradually redshifts with decreasing Si pillar width (dashed line). This energy shift is particularly strong for the InGaAs emission from the two smallest pillar sizes. The top (001) QWs on the $3 \times 3 \mu \mathrm{m}^{2} \mathrm{Ge} / \mathrm{Si}$ pillars emit at
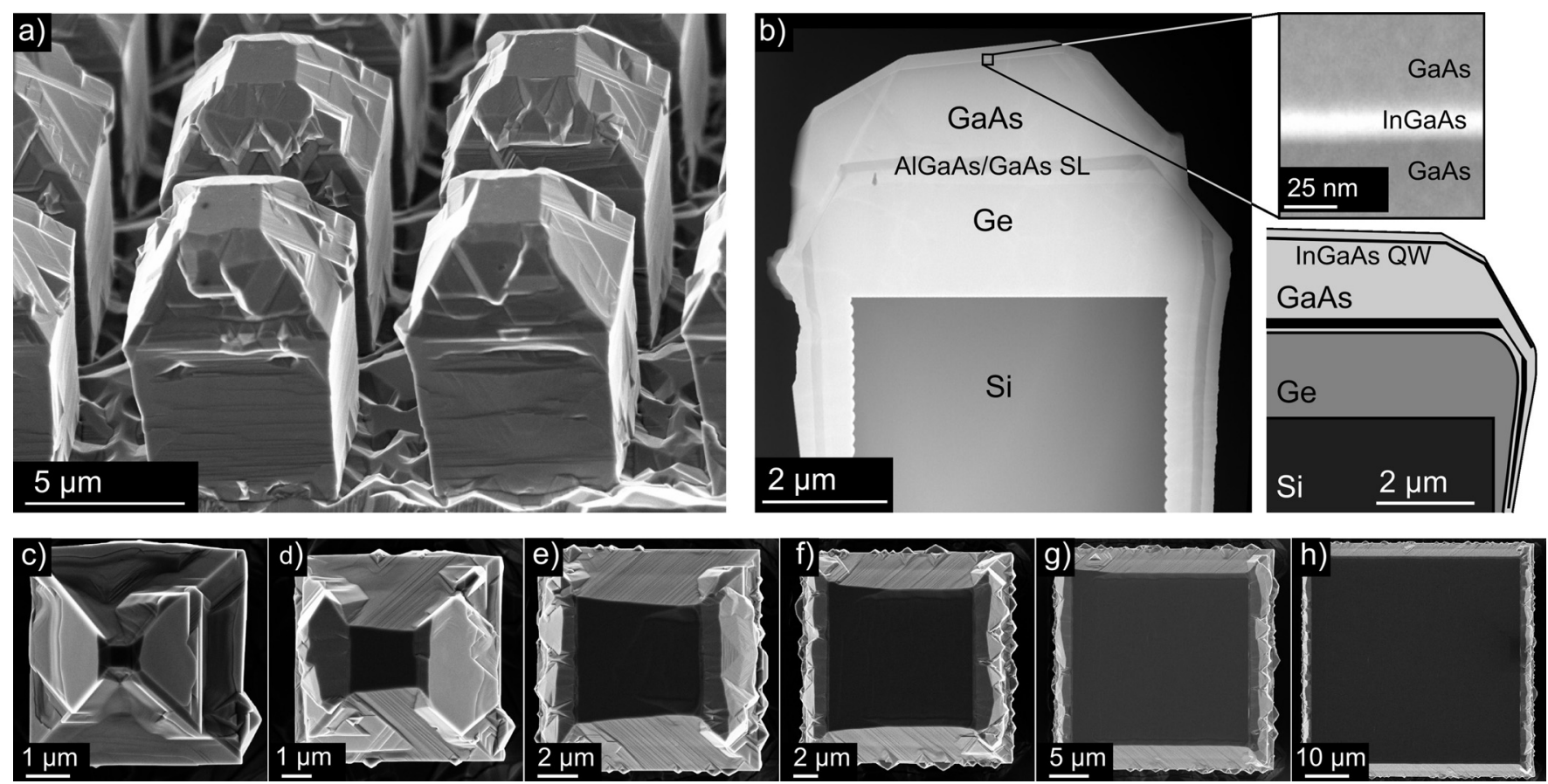

FIG. 1. Perspective view SEM images of GaAs/Ge truncated pyramids on $8 \mu \mathrm{m}$ tall and $5 \mu \mathrm{m}$ wide Si pillars (a). STEM cross section and schematic view of the structural build-up showing the InGaAs QW (b). Detailed view of the GaAs top surface grown on 3, 5, 9, 15, 25, and 50 $\mu$ m wide square Si pillars, respectively (c)-(h). 


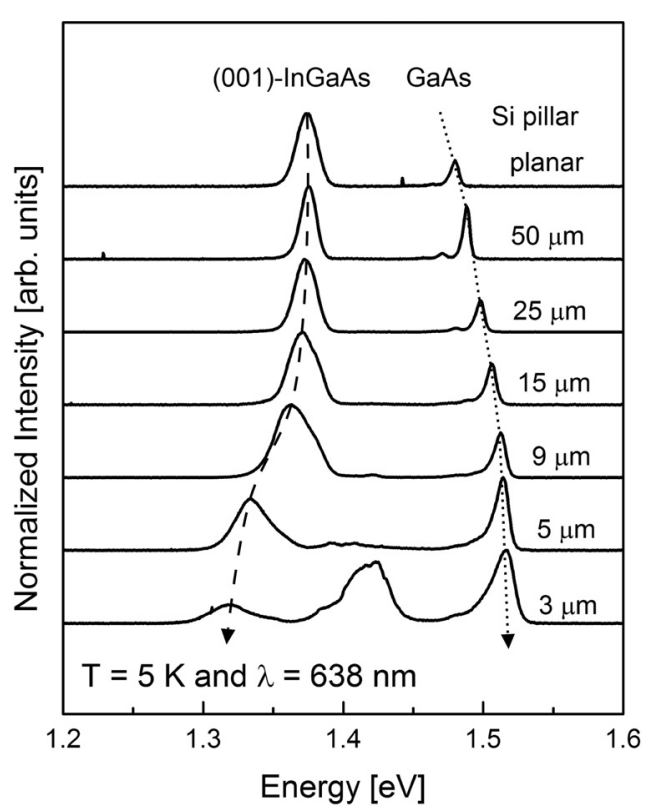

FIG. 2. Photoluminescence spectra (at $5 \mathrm{~K}$ ) of InGaAs/GaAs QWs on differently sized $\mathrm{Ge} / \mathrm{Si}$ pillars, as measured by exciting on the top (001) facet. The InGaAs peak redshifts with decreasing pillar size, whereas the GaAs peak blueshifts.

$1.313 \mathrm{eV}$, whereas those on $50 \times 50 \mu \mathrm{m}^{2} \mathrm{Ge} / \mathrm{Si}$ pillars emit at $1.375 \mathrm{eV}$, which amounts to a difference of $62 \mathrm{meV}$. In contrast to the InGaAs peak, the GaAs peak strongly blueshifts with decreasing pillar size (dotted line), indicating increasing elastic relaxation of the thermal strain induced by the mismatch of the thermal expansion coefficients between the $\mathrm{GaAs} / \mathrm{Ge}$ epilayer and the Si substrate.

Given the blueshift of the GaAs peak, the redshift of the InGaAs peak is even more striking since the QW is expected to experience the same amount of thermal strain as the surrounding GaAs. The broad intermediate PL peaks (energy of $\sim 1.42 \mathrm{eV}$ ) in the 3, 5, and $9 \mu \mathrm{m}$ spectra are attributed to InGaAs QW emission from the inclined surfaces. PL signal from those facets is related to two concomitant reasons. First, the laser spot size is comparable or even larger than the central (001) area, such that charge carriers are also photogenerated in the slanted facets. Second, electron-hole pairs photo-generated in the (001) area can diffuse and recombine in the $\{111\}$ and $\{113\}$ facets. However, QW emission from inclined surfaces showed irreproducible behavior as a function of excitation position, and in terms of energy. We attribute this behavior to the rough morphology of those surfaces leading to an inhomogeneous InGaAs QW. The low intensity PL peak next to the GaAs could be assigned to a free electron neutral acceptor transition $\left(\mathrm{e}-\mathrm{C}^{0}\right)$ due to carbon impurities incorporated during the MOVPE growth. ${ }^{16}$ Furthermore, the full width at half maximum (FWHM) of the InGaAs PL peak increases with decreasing pillar size. This suggests that the homogeneity and quality of the QWs degrade with decreasing Si pillar size.

In general, the InGaAs QW emission energy is mainly determined by three parameters. First, the thickness of the QW determines the confinement energy of the bound states. Second, strain leads to a change in band gap energy due to the change of atomic spacing. Third, the exact In content highly influences the band gap energy $\left(\mathrm{E}_{\mathrm{g}}(\mathrm{InAs})=0.415 \mathrm{eV}\right.$ and $\mathrm{E}_{\mathrm{g}}(\mathrm{GaAs})=1.519 \mathrm{eV}$ at low temperatures $) .{ }^{17}$ Since the InGaAs QW emission energies do not follow the trend of the GaAs peaks, interplay of the different parameters must occur.

Figure 3 compares the calculated $\operatorname{In}_{\mathrm{x}} \mathrm{Ga}_{1-\mathrm{x}} \mathrm{As}$ QW emission energies (black lines) to the GaAs-strain corrected experimental data (symbols). The transition energies from the lowest lying confined electron state $1 \mathrm{e}$ to its corresponding heavy hole state $1 \mathrm{hh}$ are depicted for different In concentration as a function of QW thickness. The influence of strain induced by the mismatch of $\mathrm{Si}$ and GaAs/Ge thermal expansion was eliminated by correcting the InGaAs emission peaks according to the deviation of their respective GaAs peaks from relaxed GaAs. This is justified since the lattice constants of GaAs and $\mathrm{In}_{0.13} \mathrm{Ga}_{0.87}$ As only slightly differ such that a change in strain in the GaAs leads to a change in strain in the InGaAs QW by almost the same amount. This statement holds for epitaxial films below their critical thickness, which was found to be around $30 \mathrm{~nm}$ for similar InGaAs/GaAs QW structures. ${ }^{18}$

The theoretical $\mathrm{In}_{\mathrm{x}} \mathrm{Ga}_{1-\mathrm{x}}$ As transition energies (1e-1hh) as a function of QW width and In content were calculated for a coherently lattice matched rectangular $\mathrm{QW}$ by solving the Schrödinger equation in the effective mass approximation ${ }^{19}$ using an open-source software ${ }^{20}$ and applying material parameters published by Vurgaftman et al. ${ }^{21}$ The calculated transition energies decrease with increasing In content, as expected from the band gap energies of the binary materials. Likewise, an increase of QW thickness decreases transition energies as a result of smaller confinement, which turns in a decrease of the energies of the bound states in the QW. The fact that the measured emission energy corresponding to a $10 \mathrm{~nm} \mathrm{In}_{0.13} \mathrm{Ga}_{0.87} \mathrm{As}$ QW in planar GaAs/Ge lies quite close to the calculated $\mathrm{In}_{0.13} \mathrm{Ga}_{0.87} \mathrm{As}$ line supports the correctness of the reasoning.

Comparing the measured emission energies with the calculated theoretical values, it becomes evident that the observed Si pillar size dependent shift in InGaAs emission energies (Figure 2) must be driven by a significant change in In concentration $(\sim 16 \%, \sim 20 \%, \sim 21 \%$ for InGaAs/GaAs on 15,5 , and $3 \mu \mathrm{m}$ wide Si pillars, respectively). We explain this observation by enhanced In diffusion from the inclined

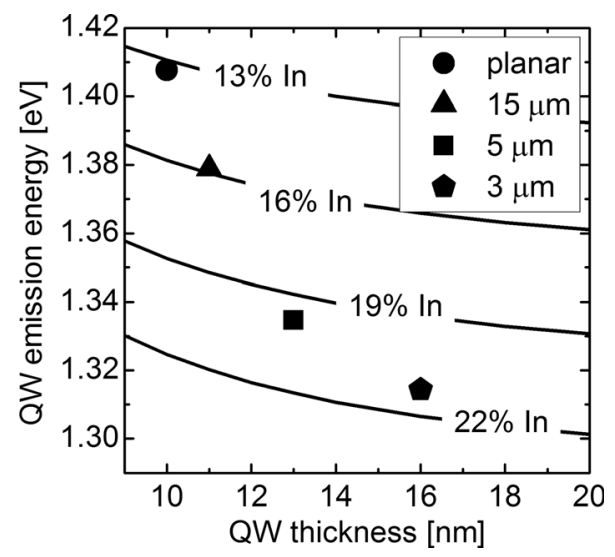

FIG. 3. Calculated emission energies vs QW thickness for InGaAs/GaAs QWs of different In concentration (black lines), and strain-corrected experimental results (symbols). 


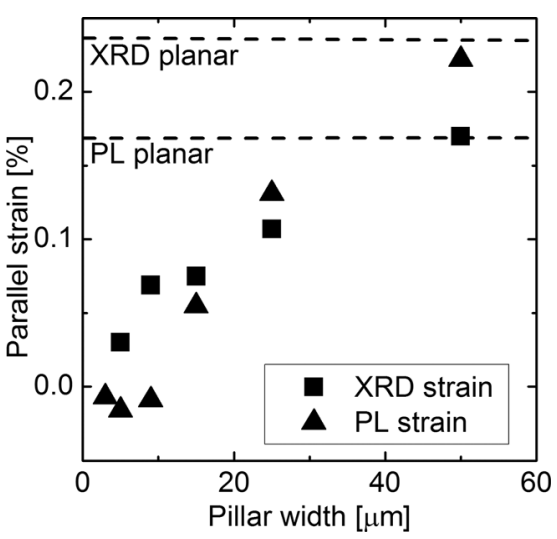

FIG. 4. Comparison of GaAs strain parallel to the wafer surface, as measured by PL and XRD versus pillar dimensions. The XRD strain evolution is flatter than that measured by PL.

facets to the top facet during growth. This explanation also accounts for the increased QW thickness for GaAs/Ge crystals on small Si pillars. Similar behavior of In adatom migration from the $\{111\}$ to the (001) GaAs facet was found in MBE growth studies. ${ }^{14}$ Correspondingly, the top surface to inclined surface ratio of the GaAs crystals strongly affects the In concentration in the top (001) QW. The increase of the InGaAs FWHM with decreasing Si pillar size (Figure 2), indicating deterioration of QW quality, is explained by the impact of the diffusion from the inclined surfaces on the growth homogeneity in [001] direction. This effect becomes stronger when the Si pillar's size is smaller because of a decreasing top to inclined facets surface ratio. Likewise, the decreasing InGaAs/GaAs PL peak intensity ratio suggests that non-radiative recombination mechanisms in the $\mathrm{QW}$ become stronger when the Si pillar is smaller. Again, this indicates a reduction of QW quality of the smaller structures.

\section{X-ray diffraction}

The Si pillar size dependent release of the strain in the GaAs crystals induced by the mismatch of thermal expansion coefficients was cross-checked with XRD as shown in Figure 4. The tensile strain in the GaAs crystals gradually decreases with the Si pillar width implying compliant elastic strain release. The small discrepancy between the evolutions of strain versus Si pillar width of the two different methods is explained by their different space and depth sensitivities. While the X-ray spot size was of the order of $1 \mathrm{~mm}$, the laser beam spot was $\sim 2 \mu \mathrm{m}$ and has a comparably small penetration depth $\left(0.27\right.$ vs $29 \mu \mathrm{m}$ in GaAs). ${ }^{22,23}$ Therefore, the strain calculated from XRD measurements is averaged over the entire volume of several crystals, leading to a smaller slope of the strain versus Si pillar width dependence.

\section{CONCLUSION}

In summary, we have studied a novel material structure made of GaAs/Ge crystals with InGaAs/GaAs QWs on $\mu \mathrm{m}$ sized square Si pillars. Such structures would allow for the monolithic integration of GaAs-based quantum emitters on $\mathrm{Si}$ via a Ge buffer layer. The crystals form truncated pyramids where the ratio of inclined to top facet area depends on the Si pillar size. PL spectroscopy in combination with STEM results reveals that the In concentration in the QWs increases with decreasing Si pillar size. This observation is attributed to pronounced In diffusion from the inclined facets to the top (001) facet during growth. These results will help to improve and contribute to the development of quantum optoelectronic devices and are encouraging for future experiments, such as the incorporation of quantum dots. ${ }^{15}$

\section{ACKNOWLEDGMENTS}

We acknowledge E. Müller, I. Prieto, E. Gini, and B. Batlogg for valuable scientific discussions and the FIRST center for micro- and nano-science and ScopeM scientific center for optical and electron microscopy for making available their infrastructure. This work was supported by ETH Research Grant ETH-20 11-2, and the Sinergia project NOVIPIX of the Swiss National Science Foundation.

${ }^{1}$ Y. B. Bolkhovityanov and O. P. Pchelyakov, Rev. Top. Probl. 51, 437 (2008).

${ }^{2}$ A. G. Taboada, T. Kreiliger, C. V. Falub, F. Isa, M. Salvalaglio, L. Wewior, D. Fuster, M. Richter, E. Uccelli, P. Niedermann, A. Neels, F. Mancarella, B. Alén, L. Miglio, A. Dommann, G. Isella, and H. von Känel, Appl. Phys. Lett. 104, 022112 (2014).

${ }^{3}$ A. Hierro, M. Hansen, J. J. Boeckl, L. Zhao, J. S. Speck, U. K. Mishra, S. P. DenBaars, and S. A. Ringel, Phys. Status Solidi B 228, 937 (2001).

${ }^{4}$ T. Li, M. Mastro, A. Dadgar, A. Ringel, and T. Grassman, III-V Compound Semiconductors. Integration with Silicon-Based Microelectronics (CRC Press, Boca Raton, 2011).

${ }^{5}$ R. M. Sieg, S. A. Ringel, S. M. Ting, E. A. Fitzgerald, and R. N. Sacks, J. Electron. Mater. 27, 900 (1998).

${ }^{6}$ E. Fitzgerald and N. Chand, J. Electron. Mater. 20, 839 (1991).

${ }^{7}$ S. Ren, Ph.D. thesis, Stanford University, Stanford, 2011.

${ }^{8}$ C. V. Falub, H. von Känel, F. Isa, R. Bergamaschini, A. Marzegalli, D. Chrastina, G. Isella, E. Müller, P. Niedermann, and L. Miglio, Science 335, 1330 (2012).

${ }^{9}$ C. Rosenblad, H. von Känel, M. Kummer, A. Dommann, and E. Müller, Appl. Phys. Lett. 76, 427 (2000).

${ }^{10}$ A. Marzegalli, F. Isa, H. Groiss, E. Müller, C. V. Falub, A. G. Taboada, P. Niedermann, G. Isella, F. Schäffler, F. Montalenti, H. Von Känel, and L. Miglio, Adv. Mater. 25, 4408 (2013).

${ }^{11}$ F. Laermer and A. Schilp, U.S. patent No. 5,501,893 (26 March 1996).

${ }^{12}$ W. T. Tsang and A. Y. Cho, Appl. Phys. Lett. 30, 293 (1977).

${ }^{13}$ S. Koshiba, H. Noge, H. Akiyama, T. Inoshita, Y. Nakamura, A. Shimizu, Y. Nagamune, M. Tsuchiya, H. Kano, H. Sakaki, and K. Wada, Appl. Phys. Lett. 64, 363 (1994).

${ }^{14}$ D. J. Arent, S. Nilsson, Y. D. Galeuchet, H. P. Meier, and W. Walter, Appl. Phys. Lett. 55, 2611 (1989).

${ }^{15}$ P. S. Wong, B. L. Liang, V. G. Dorogan, A. R. Albrecht, J. Tatebayashi, X. He, N. Nuntawong, Y. I. Mazur, G. J. Salamo, S. R. J. Brueck, and D. L. Huffaker, Nanotechnology 19, 435710 (2008).

${ }^{16}$ J. Z. Li, J. Bai, J. M. Hydrick, J. S. Park, C. Major, M. Carroll, J. G. Fiorenza, and A. Lochtefeld, J. Cryst. Growth 311, 3133 (2009).

${ }^{17}$ K.-H. Goetz, D. Bimberg, H. Juürgensen, J. Selders, A. V. Solomonov, G. F. Glinskii, and M. Razeghi, J. Appl. Phys. 54, 4543 (1983).

${ }^{18}$ J. Mantz, C. Shieh, H. Lee, D. Ackley, and R. Engelmann, Electron. Lett. 25, 1226 (1989)

${ }^{19}$ T. Ihn, Semiconductor Nanostructures, Quantum States and Electronic Transport (Oxford University Press, New York, 2010).

${ }^{20} \mathrm{M}$. Wesolowski, EPITAXYproject Epitaxy Supporting Software (Kaliska Str. 26, 87-800 Wloclawek, Poland, 2014).

${ }^{21}$ I. Vurgaftman, J. R. Meyer, and L. R. Ram-Mohan, J. Appl. Phys. 89, 5815 (2001).

${ }^{22}$ D. Palik, Handbook of Optical Constants of Solids (Academic Press, New York, 1985).

${ }^{23}$ C. T. Chantler, J. Phys. Chem. Ref. Data 24, 71 (1995). 